\title{
Scheduling and Codeword Length Optimization in Time Varying Wireless Networks
}

\author{
Mehdi Ansari Sadrabadi, Alireza Bayesteh and Amir K. Khandani \\ Coding \& Signal Transmission Laboratory(www.cst.uwaterloo.ca) \\ Dept. of Elec. and Comp. Eng., University of Waterloo \\ Waterloo, ON, Canada, N2L 3G1 \\ Tel: 519-884-8552, Fax: 519-888-4338 \\ e-mail: \{mehdi, alireza, khandani\}@ cst.uwaterloo.ca
}

\begin{abstract}
In this paper, a downlink scenario in which a single-antenna base station communicates with $K$ single antenna users, over a time-correlated fading channel, is considered. It is assumed that channel state information is perfectly known at each receiver, while the statistical characteristics of the fading process and the fading gain at the beginning of each frame are known to the transmitter. By evaluating the random coding error exponent of the time-correlated fading channel, it is shown that there is an optimal codeword length which maximizes the throughput. The throughput of the conventional scheduling that transmits to the user with the maximum signal to noise ratio is examined using both fixed length codewords and variable length codewords. Although optimizing the codeword length improves the performance, it is shown that using the conventional scheduling, a gap of $\Omega(\sqrt{\log \log \log K})$ exists between the achievable throughput and the maximum possible throughput of the system. A simple scheduling that considers both the signal to noise ratio and the channel time variation is proposed. It is shown that by using this scheduling, the gap between the achievable throughput and the maximum throughput of the system approaches zero.
\end{abstract}

\section{Index Terms}

Downlink scheduling, multiuser diversity, Rayleigh fading, time varying channels.

\section{INTRODUCTION}

In wireless networks, diversity is a means to combat the time varying nature of the communication link. Conventional diversity techniques over point-to-point links, such as spatial diversity and frequency diversity are widely used and offer performance improvements. In multiuser wireless systems, there exists another form of diversity, called multiuser diversity [1]. In a broadcast channel where users have independent fading and feed back their signal to noise ratio (SNR) to the base station (BS), system throughput is maximized by transmitting to the user with the strongest SNR [1], [2].

Multiuser diversity was introduced first by Knopp and Humblet [3]. It is shown that the optimal transmission strategy in the uplink of multiuser systems using power control is to only let the user with the largest SNR transmit. A similar result is shown to be valid for the downlink [4]. Multiuser diversity underlies much of the recent works for downlink scheduling [5]-[8] as in Qualcomm's high data rate (HDR) system [9], [10]. In [8], [11], the opportunistic scheduling is based on the highest data rate which can be reliably transmitted to each user. Distributed scheduling is proposed in an uplink scenario, where full channel state information (CSI) is not required at the transmitter [12], [13]. Multiuser diversity has also been studied in the context of multiple antenna systems [1], [14] and ad-hoc networks [15].

In wireless networks, the rate of channel variations is characterized by maximum Doppler frequency which is proportional to the velocity. Utilizing multiuser diversity in such environments needs to be revisited since the throughput depends not only on the received SNR, but also on how fast the channel varies over time.

In this paper, we consider a broadcast channel in which a BS transmits data to a large number of users in a time-correlated flat fading environment. It is assumed that CSI is perfectly known to the receivers, while BS only knows the statistical characteristics of the fading process for all the users (which is assumed to be 
constant during a long period). Moreover, each user feeds back its channel gain to the BS at the beginning of each frame. Based on this information, BS selects a single user for transmission in each frame, in order to maximize the throughput. For the case of Additive White Gaussian Noise (AWGN) or block fading, it is well known that increasing the codeword length results in improving the achievable throughput. However, in a time varying channel, it is not possible to obtain arbitrary small error probabilities by increasing the codeword length. In fact, increasing the codeword length also results in increasing the fading fluctuations over the frame, and consequently, the throughput will decrease. Therefore, it is of interest to find the optimum codeword length which maximizes the throughput.

In this paper, a downlink scenario in which a single-antenna base station communicates with $K$ single antenna users, over a time-correlated fading channel, is considered. We analyze different user selection strategies; i) the BS transmits data to the user with the strongest SNR using fixed length codewords (conventional multiuser scheduling), ii) the BS transmits data to the user with the strongest SNR using variable length codewords, and iii) the BS transmits data to the user that achieves the maximum throughput using variable length codewords. We show that in all cases the achievable throughput scales as $\log \log K$. Moreover, in cases (i) and (ii), the gap between the achievable throughput and the maximum throughput scales as $\sqrt{\log \log \log K}$, while in case (iii), this gap approaches zero.

The rest of the paper is organized as follows. In Section $\amalg$, the model of time-correlated fading channel is described. In Section III, different user selection strategies are discussed and the corresponding throughput of the system is derived for each strategy, for $K \rightarrow \infty$. Finally, in Section IV, we conclude the paper.

Throughout this paper, $\mathbb{E}\{$.$\} and \operatorname{var}\{$.$\} represents the expectation and variance, respectively, "log" is$ used for the natural logarithm, and rate is expressed in nats. For given functions $f(N)$ and $g(N), f(N)=$ $O(g(N))$ is equivalent to $\lim _{N \rightarrow \infty}\left|\frac{f(N)}{g(N)}\right|<\infty, f(N)=o(g(N))$ is equivalent to $\lim _{N \rightarrow \infty}\left|\frac{f(N)}{g(N)}\right|=$ $0, f(N)=\omega(g(N))$ is equivalent to $\lim _{N \rightarrow \infty} \frac{f(N)}{g(N)}=\infty$, and $f(N)=\Omega(g(N))$ is equivalent to $\lim _{N \rightarrow \infty} \frac{f(N)}{g(N)}=c$, where $0<c<\infty$.

\section{SYSTEM MODEL}

The channel of any given user is modeled as a time-correlated fading process. It is assumed that the channel gain is constant over each channel use (symbol) and varies from symbol to symbol, following a Markovian random process. Assume that the fading gain of $k^{\text {th }}$ user is $\boldsymbol{h}_{k}=\left[h_{1, k}, \ldots, h_{N_{k}, k}\right]^{T}$ where $h_{i, k}, 1 \leq i \leq N_{k}$ are complex Gaussian random variables with zero mean and unit variance and $N_{k}$ is the codeword length of the $k^{\text {th }}$ user. The received signal for the $k^{\text {th }}$ user is given by

$$
\boldsymbol{r}_{k}=\boldsymbol{S}_{k} \boldsymbol{h}_{k}+\boldsymbol{n}_{k},
$$

where $\boldsymbol{S}_{k}=\operatorname{diag}\left(s_{1, k}, s_{2, k}, \ldots, s_{N_{k}, k}\right)$ is the transmitted codeword with the power constraint $11 \mathbb{E}\left\{\left|s_{i, k}\right|^{2}\right\} \leq$ $P$, and $\boldsymbol{n}_{k}$ is AWGN with zero mean and covariance matrix $\boldsymbol{I}$. Assume that $h_{0, k}$ is the fading gain at the time instant before $\boldsymbol{S}_{k}$ is transmitted. The sequence $u_{i, k}=\left|h_{i, k}\right|, 0 \leq i \leq N_{k}$, is assumed to be a stationary ergodic chain with the following probability density function [16]:

$$
\begin{gathered}
f_{u_{0, k}}(u)= \begin{cases}2 u e^{-u^{2}} & u \geq 0 \\
0 & \text { otherwise }\end{cases} \\
f\left(u_{1, k}, u_{2, k}, \cdots, u_{N_{k}, k} \mid u_{0, k}\right)=\prod_{i=1}^{N_{k}} q_{k}\left(u_{i, k} \mid u_{i-1, k}\right),
\end{gathered}
$$

where,

$$
q_{k}(u \mid v)= \begin{cases}\frac{2 u}{1-\alpha_{k}^{2}} \exp \left(-\frac{u^{2}+\alpha_{k}^{2} v^{2}}{1-\alpha_{k}^{2}}\right) \mathcal{I}_{0}\left(\frac{2 \alpha_{k} u v}{1-\alpha_{k}^{2}}\right) & u \geq 0 \\ 0 & \text { otherwise }\end{cases}
$$

\footnotetext{
${ }^{1}$ Obviously, for maximizing the throughput, the power constraint translates to $\mathbb{E}\left\{\left|s_{i, k}\right|^{2}\right\}=P$.
} 
in which $0<\alpha_{k}<1$ describes the channel correlation coefficient of the $k^{\text {th }}$ user. It is assumed that $\alpha_{k}, 1 \leq k \leq K$, are i.i.d. random variables with uniform distribution which remain fixed during the entire transmission, and $\mathcal{I}_{0}($.$) denotes the modified Bessel function of order zero. It is assumed that CSI$ is perfectly known at each receiver, while the statistical characteristics of the fading process and $u_{0, k}$, $1 \leq k \leq K$ are known to the transmitter.

\section{Throughrut ANALYSIS}

In this section, we derive the achievable throughput of the system in the asymptotic case of $K \rightarrow \infty$. We define the $k^{\text {th }}$ user's throughput per channel use, denoted by $T_{k}$, as

$$
T_{k} \triangleq R_{k}\left(1-p_{e}(k)\right)
$$

where $R_{k}$ is the transmitted rate per channel use and $p_{e}(k)$ is the frame error probability for this user. Using the concept of random coding error exponent [17], $p_{e}(k)$ can be upper-bounded as

$$
p_{e}(k) \leq \inf _{0 \leq \rho \leq 1} e^{-N\left(E_{k}(\rho)-\rho R_{k}\right)} .
$$

For simplicity of analysis, we use this upper-bound in evaluating the throughput. This bound is tight for rates close to the capacity as used in [18]-[20].

Assuming $s_{i, k}, 1 \leq i \leq N_{k}$, are Gaussian and i.i.d., it is shown that the random coding error exponent for the $k^{\text {th }}$ user, $E_{k}(\rho)$, is given by [20],

$$
E_{k}(\rho)=-\frac{1}{N_{k}} \log \mathbb{E}_{\boldsymbol{u}_{k}}\left\{\prod_{i=1}^{N_{k}}\left(\frac{1}{1+\frac{P}{1+\rho} u_{i, k}^{2}}\right)^{\rho}\right\} .
$$

where $\boldsymbol{u}_{k}=\left[u_{1, k}, \ldots, u_{N_{k}, k}\right]$.

In the following, we assume that $u_{0, k} \gg 1$. Since in strategies introduced in this work, a user is selected if the corresponding initial fading gain is maximum or above a certain threshold, this assumption is valid when the number of users is large.

Theorem 1 For the channel model described in the previous section, and assuming $u_{0, k}$ is known, we have

$$
E_{k}(\rho)=\frac{1}{N_{k}} \sum_{i=1}^{N_{k}} \rho \log \left(1+\frac{P u_{0, k}^{2} \alpha_{k}^{2 i}}{(1+\rho)}\right)+O\left(\frac{1}{\sqrt{u_{0, k}}}\right)-O\left(e^{-u_{0, k}^{2}}\right) .
$$

Proof: See Appendix A. 
Minimizing (5) is equivalent to maximizing $E_{k}(\rho)-\rho R_{k}$. Noting (7), we have

$$
\begin{aligned}
E_{k}(\rho)-\rho R_{k} & =\frac{1}{N_{k}} \sum_{i=1}^{N_{k}} \rho \log \left(1+\frac{P u_{0, k}^{2} \alpha_{k}^{2 i}}{(1+\rho)}\right)-\rho R_{k}+O\left(\frac{1}{\sqrt{u_{0, k}}}\right)-O\left(e^{-u_{0, k}^{2}}\right) \\
& =\frac{1}{N_{k}} \sum_{i=1}^{N_{k}} \rho \log \left(\frac{P u_{0, k}^{2} \alpha_{k}^{2 i}}{(1+\rho)}\right)-\rho R_{k}+\frac{1}{N_{k}} \sum_{i=1}^{N_{k}} \rho \log \left(1+\frac{(1+\rho)}{P u_{0, k}^{2} \alpha_{k}^{2 i}}\right) \\
& +O\left(\frac{1}{\sqrt{u_{0, k}}}\right)-O\left(e^{-u_{0, k}^{2}}\right) \\
& =\frac{\rho}{N_{k}} \sum_{i=1}^{N_{k}}\left(\log \left(P u_{0, k}^{2}\right)+2 i \log \left(\alpha_{k}\right)-\log (1+\rho)\right)-\rho R_{k} \\
& +\frac{1}{N_{k}} \sum_{i=1}^{N_{k}} O\left(\frac{1}{u_{0, k}^{2}}\right)+O\left(\frac{1}{\sqrt{u_{0, k}}}\right)-O\left(e^{-u_{0, k}^{2}}\right) \\
& =\rho\left[\log \left(P u_{0, k}^{2}\right)+\left(N_{k}+1\right) \log \left(\alpha_{k}\right)-\log (\rho+1)-R_{k}\right] \\
& +O\left(\frac{1}{\sqrt{u_{0, k}}}\right)-O\left(e^{-u_{0, k}^{2}}\right)
\end{aligned}
$$

It is easy to show that $\rho_{k}^{\text {opt }}$ which maximizes (8) for large values of $u_{0, k}$ is

$$
\begin{array}{r}
\log \left(1+\rho_{k}^{\mathrm{opt}}\right)+\frac{\rho_{k}^{\mathrm{opt}}}{1+\rho_{k}^{\mathrm{opt}}}=\beta_{k}, \quad \beta_{k}<\log (2)+\frac{1}{2} \\
\rho_{k}^{\mathrm{opt}}=1, \quad \beta_{k} \geq \log (2)+\frac{1}{2}
\end{array}
$$

where

$$
\beta_{k}=\log \left(P u_{0, k}^{2}\right)+\left(N_{k}+1\right) \log \left(\alpha_{k}\right)-R_{k} .
$$

Using (4), (5), (7) and (8), we have

$$
\begin{aligned}
T_{k} & =R_{k}\left[1-e^{-N_{k}\left(E_{k}\left(\rho_{k}^{\mathrm{opt}}\right)-\rho_{k}^{\mathrm{opt}} R_{k}\right)}\right] \\
& =R_{k}\left[1-e^{-\rho_{k}^{\mathrm{opt}} N_{k}\left(\log \left(P u_{0, k}^{2}\right)+\left(N_{k}+1\right) \log \left(\alpha_{k}\right)-\log \left(\rho_{k}^{\mathrm{opt}}+1\right)-R_{k}\right)}\right] .
\end{aligned}
$$

It is easy to show that $T_{k}$ is a concave function of variables $R_{k}$ and $N_{k}$, and the values of $R_{k}$ and $N_{k}$ which maximize the throughput $\left(R_{k}^{\mathrm{opt}}\right.$ and $\left.N_{k}^{\mathrm{opt}}\right)$ satisfy the following equations:

$$
\begin{gathered}
R_{k}^{\mathrm{opt}}=\log \left(P u_{0, k}^{2}\right)+\left(2 N_{k}^{\mathrm{opt}}+1\right) \log \left(\alpha_{k}\right)-\log \left(\rho_{k}^{\mathrm{opt}}+1\right), \\
N_{k}^{\mathrm{opt}}=\sqrt{\frac{\log \left(1+\rho_{k}^{\mathrm{opt}} N_{k}^{\mathrm{opt}} R_{k}^{\mathrm{opt}}\right)}{\rho_{k}^{\mathrm{opt}} \log \left(\alpha_{k}^{-1}\right)}} .
\end{gathered}
$$

It follows that $N_{k}^{\text {opt }} \rightarrow \infty$ and $R_{k}^{\text {opt }} \rightarrow \infty$ as $u_{0, k} \rightarrow \infty$. Using (12) and (13), (11) can be re-written as follows:

$$
T_{k}=\left(\log \left(\frac{P u_{0, k}^{2}}{\rho_{k}^{\mathrm{opt}}+1}\right)+\left(2 N_{k}^{\mathrm{opt}}+1\right) \log \left(\alpha_{k}\right)\right)\left(1-\frac{1}{1+\rho_{k}^{\mathrm{opt}} N_{k}^{\mathrm{opt}} R_{k}^{\mathrm{opt}}}\right)
$$

Substituting (12) in (10), we have

$$
\beta_{k}=N_{k}^{\mathrm{opt}} \log \left(\alpha_{k}^{-1}\right)+\log \left(\rho_{k}^{\mathrm{opt}}+1\right) .
$$

${ }^{2}$ Note that we have relaxed the condition of $N_{k}$ being integer. However, since the optimizing $N_{k}$ tends to infinity as $K \rightarrow \infty$, this assumption does not affect the result. 
From (9) and (15), it is concluded that

$$
\rho_{k}^{\mathrm{opt}}= \begin{cases}\frac{N_{k}^{\mathrm{opt}} \log \left(\alpha_{k}^{-1}\right)}{1-N_{k}^{\mathrm{opt}} \log \left(\alpha_{k}^{-1}\right)} & N_{k}^{\mathrm{opt}} \log \left(\alpha_{k}^{-1}\right)<\frac{1}{2} \\ 1 & N_{k}^{\mathrm{opt}} \log \left(\alpha_{k}^{-1}\right) \geq \frac{1}{2}\end{cases}
$$

Noting (12) and (16), for $\alpha_{k}=1$, we have $\rho_{k}^{\text {opt }}=0$ and $R_{k}^{\text {opt }}=\log \left(P u_{0, k}^{2}\right)$ which corresponds to the capacity of a quasi-static fading channel (for large values of channel gain $u_{0, k}^{2}$ ).

In the following, we obtain the asymptotic throughput of the $k^{\text {th }}$ user. Since there are two regions for $\rho_{k}^{\text {opt }}$ as shown in (16), we calculate the closed form formula of the throughputs of these two cases separately.

- $\left(\rho_{k}^{\mathrm{opt}}=1\right)$ : The corresponding asymptotic throughput is obtained by substituting (12) and (13) in (11) as follows:

$$
\begin{aligned}
T_{k}= & \log \left(\frac{P u_{0, k}^{2}}{2}\right)-2 \sqrt{\log \left(\alpha_{k}^{-1}\right) \log \log \left(\frac{P u_{0, k}^{2}}{2}\right)} \times \\
& \left(1+O\left(\frac{\log \log \log \left(u_{0, k}\right)}{\log \log \left(u_{0, k}\right)}\right)\right) .
\end{aligned}
$$

From (13), the optimum codeword length scales as follows:

$$
N_{k}^{\text {opt }} \sim \sqrt{\frac{\log \log \left(P u_{0, k}^{2}\right)}{\log \alpha^{-1}}} .
$$

Note that if $\alpha_{k}$ is fixed and $\alpha_{k} \neq 1$, then $N_{k}^{\text {opt }} \log \left(\alpha_{k}^{-1}\right) \geq \frac{1}{2}$ for large values of $u_{0, k}$ and (17) is appliable.

- $\left(\rho_{k}^{\mathrm{opt}}<1\right)$ : We limit the calculation of the throughput to the assumption of $N_{k}^{\mathrm{opt}} \log \left(\alpha_{k}^{-1}\right) \sim o(1)$ to be able to derive the following closed form formula by using (14) and (16):

$$
\begin{aligned}
T_{k}= & \log \left(P u_{0, k}^{2}\right)-2 \sqrt[3]{\log \left(\alpha_{k}^{-1}\right) \log \log \left(P u_{0, k}^{2}\right)} \times \\
& \left(1+O\left(\frac{\log \log \log \left(u_{0, k}\right)}{\log \log \left(u_{0, k}\right)}\right)\right)-o(1) .
\end{aligned}
$$

The corresponding optimum codeword length scales as follows:

$$
N_{k}^{\mathrm{opt}} \sim \sqrt[3]{\frac{\log \log \left(P u_{0, k}^{2}\right)}{\left(\log \alpha^{-1}\right)^{2}}} .
$$

As we will see later, this special case is sufficient to accomplish the calculations required in III-C. From the above equations, it is concluded that the throughput not only depends on the initial fading gain, $u_{0, k}$, but also on the fading correlation coefficient. Moreover, the throughput is an increasing function of the channel correlation coefficient.

In the following, we introduce three scheduling strategies in order to maximize the throughput; i) Traditional scheduling in which the user with the largest channel gain is selected (SNR-based scheduling) and the codeword length is assumed to be fixed. ii) SNR-based scheduling with optimized codeword length regarding the channel condition of the selected user, and iii) Scheduling which exploits both the channel gain and the channel correlation coefficient of the users. The asymptotic throughput of the system is derived under each strategy for $K \rightarrow \infty$. 


\section{A. Strategy I: SNR-based scheduling with fixed codeword length}

The BS transmits to the user with the maximum initial fading gain. The codeword length of all users is fixed, i.e., $N_{1}=N_{2}=\cdots=N_{K}=N$. The codeword length $N$ is selected such that the throughput of the system is maximized. The BS adapts the data rate to maximize the throughput of the selected user.

Theorem 2 The asymptotic throughput of the system under Strategy I scales as

$$
\overline{\mathcal{T}}_{1} \sim \log \left(\frac{P \log K}{2}\right)-2 \sqrt{\mathbb{E}\left\{\log \left(\alpha^{-1}\right)\right\} \log \log \log K},
$$

as $K \rightarrow \infty$.

For simplicity of notation, we define $v_{k} \triangleq u_{0, k}^{2}$. Let $v=\max _{1 \leq k \leq K} v_{k}$ and $\alpha$ be the corresponding correlation coefficient of the selected user. Setting the derivative of (11) with respect to $R_{k}$ to zero, we find the rate of the selected user and the corresponding throughput in terms of $v$ and $\alpha$ as follows ${ }^{3}$ :

$$
\begin{aligned}
R= & \log \left(\frac{P v}{1+\rho^{\mathrm{opt}}}\right)+(N+1) \log (\alpha)-\frac{\log \left(1+\rho^{\mathrm{opt}} N R\right)}{\rho^{\mathrm{opt}} N} \\
\mathcal{T}_{1}(v, \alpha)= & {\left[\log \left(\frac{P v}{1+\rho^{\mathrm{opt}}}\right)+(N+1) \log (\alpha)-\frac{\log \left(1+\rho^{\mathrm{opt}} N R\right)}{\rho^{\mathrm{opt}} N}\right] \times } \\
& {\left[1-\frac{1}{1+\rho^{\mathrm{opt}} N R}\right], }
\end{aligned}
$$

where noting (9), $\rho^{\mathrm{opt}}$ is determined as follows:

$$
\begin{aligned}
\log \left(1+\rho^{\mathrm{opt}}\right)+\frac{\rho^{\mathrm{opt}}}{1+\rho^{\mathrm{opt}}} & =\beta, \quad \beta<\log (2)+\frac{1}{2} \\
\rho^{\mathrm{opt}} & =1, \quad \beta \geq \log (2)+\frac{1}{2}
\end{aligned}
$$

Using (10) and (22), we have

$$
\beta=\log \left(1+\rho^{\mathrm{opt}}\right)+\frac{\log \left(1+\rho^{\mathrm{opt}} N R\right)}{\rho^{\mathrm{opt}} N} .
$$

Let us define $R^{*}$ and event $\mathcal{A}$ as follows:

$$
\begin{gathered}
R^{*} \stackrel{\triangleq}{\triangleq} \log (P v)+(N+1) \log (\alpha) \\
\stackrel{22,25]}{=} R+\beta, \\
\mathcal{A} \equiv\left\{R^{*}>\frac{1}{2} \log \log K\right\},
\end{gathered}
$$

In the following, we derive upper-bounds for the throughput of the system in terms of $R^{*}$ and $\mathcal{A}$ which we use later in Lemma 1 and Lemma 2 .

$$
\begin{aligned}
\overline{\mathcal{T}}_{1}= & \mathbb{E}\left\{\mathcal{T}_{1}(v, \alpha)\right\} \\
\stackrel{22, \sqrt{23}}{\leq} & \mathbb{E}\{R\} \\
= & \mathbb{E}\{R \mid \mathcal{A}\} \operatorname{Pr}\{\mathcal{A}\}+\mathbb{E}\left\{R \mid \mathcal{A}^{C}\right\} \operatorname{Pr}\left\{\mathcal{A}^{C}\right\} \\
\stackrel{226}{=} & \left(\mathbb{E}\left\{R^{*} \mid \mathcal{A}\right\}-\mathbb{E}\{\beta \mid \mathcal{A}\}\right) \operatorname{Pr}\{\mathcal{A}\}+\mathbb{E}\left\{R \mid \mathcal{A}^{C}\right\} \operatorname{Pr}\left\{\mathcal{A}^{C}\right\} \\
\leq & \mathbb{E}\left\{R^{*}\right\}-\mathbb{E}\{\beta \mid \mathcal{A}\} \operatorname{Pr}\{\mathcal{A}\} \\
\leq & \mathbb{E}\left\{R^{*}\right\}
\end{aligned}
$$

${ }^{3}$ We drop user index of parameters $R$ and $\rho^{\text {opt }}$ for the selected user. 
where (28) is derived by replacing $R$ with $R^{*}$, noting $R \leq R^{*}$, and $\operatorname{Pr}\{\mathcal{A}\}$ can be computed as follows:

$$
\begin{aligned}
\operatorname{Pr}\{\mathcal{A}\} & =\operatorname{Pr}\left\{\log (P v)+(N+1) \log \alpha>\frac{1}{2} \log \log K\right\} \\
& =1-\operatorname{Pr}\left\{\log (P v)+(N+1) \log \alpha \leq \frac{1}{2} \log \log K\right\} \\
& =1-\int_{0}^{\infty} \operatorname{Pr}\left\{\log \alpha<\frac{1}{N+1}\left(\frac{1}{2} \log \log K-\log (P x)\right) \mid x\right\} f_{v}(x) d x \\
& =1-\int_{0}^{\infty} F_{\alpha}\left(e^{\frac{1}{N+1}\left(\frac{1}{2} \log \log K-\log (P x)\right)}\right) f_{v}(x) d x
\end{aligned}
$$

where $f_{y}($.$) and F_{y}($.$) are probability density function and cumulative density function of random variable$ $y$, respectively. Noting that $\alpha$ has a uniform distribution, we have

$$
\begin{aligned}
\operatorname{Pr}\{\mathcal{A}\} & =1-e^{\frac{\log \log K}{2(N+1)}} \int_{0}^{\infty} e^{-\frac{\log (P x)}{N+1}} f_{v}(x) d x \\
& \sim 1-e^{-\frac{\log \log K+2 \log (P)}{2(N+1)}},
\end{aligned}
$$

where the second line follows from the fact that $v \sim \log K$, with probability one [21].

According to (24), there are two regions for $\rho^{\text {opt }}$ of the selected user. To obtain the throughput of the system, we upper-bound the throughput in these two regions in Lemma 1 and Lemma 2, respectively. Then, we derive a lower-bound for the throughput of the system in Lemma 4.

Lemma 1 Assuming $\beta<\log (2)+\frac{1}{2}$, the throughput of Strategy I is upper-bounded as follows:

$$
\overline{\mathcal{T}}_{11} \lesssim \log (P \log K)-(\log (\log \log K-2 \log (2))) \mathbb{E}\left\{\log \left(\alpha^{-1}\right)\right\} \operatorname{Pr}\{\mathcal{A}\}
$$

Proof: Using (24) and (25) and noting $\beta<\log (2)+\frac{1}{2}$, we obtain

$$
\left(\rho^{\mathrm{opt}}\right)^{-1}+\left(\rho^{\mathrm{opt}}\right)^{-2}=\frac{N}{\log \left(1+\rho^{\mathrm{opt}} N R\right)} .
$$

Noting $\rho^{\text {opt }}<1$, it follows from (33) that

$$
N>\log \left(1+\rho^{\mathrm{opt}} N R\right) .
$$

Assuming $R$ is large enough, from (33), we have

$$
\frac{\rho^{\mathrm{opt}} N R}{\log \left(1+\rho^{\mathrm{opt}} N R\right)}>2 R \Rightarrow \rho^{\mathrm{opt}} N>2 .
$$

Using (34) and (35), we can write

$$
\begin{aligned}
& N \stackrel{34}{>} \mathbb{E}\left\{\log \left(1+\rho^{\mathrm{opt}} N R\right) \mid \mathcal{A}\right\} \operatorname{Pr}\{\mathcal{A}\} \\
& \stackrel{35}{>} \mathbb{E}\{(\log (1+2 R)) \mid \mathcal{A}\} \operatorname{Pr}(\mathcal{A}) \\
& \stackrel{a}{>}(\log (\log \log K-2 \log (2))) \operatorname{Pr}\{\mathcal{A}\}
\end{aligned}
$$

where (a) results from the fact that conditioned on $\mathcal{A}$, we have $R=R^{*}-\beta>\frac{1}{2} \log \log K-\frac{1}{2}-\log (2)$. Noting that $v \sim \log K+O(\log \log K)$ with probability one [21], we can write the throughput of the system as follows:

$$
\begin{aligned}
\overline{\mathcal{T}}_{11} \stackrel{26}{ } \stackrel{29}{\leq} & \mathbb{E}\{\log (P v)+(N+1) \log (\alpha)\} \\
= & \mathbb{E}\{\log (P v)\}-(N+1) \mathbb{E}\left\{\log \left(\alpha^{-1}\right)\right\} \\
& \stackrel{36}{\lesssim} \log (P \log K)-(\log (\log \log K-2 \log (2))) \mathbb{E}\left\{\log \alpha^{-1}\right\} \operatorname{Pr}\{\mathcal{A}\} .
\end{aligned}
$$


Lemma 2 Assuming $\beta \geq \log (2)+\frac{1}{2}$, the throughput of Strategy I is upper-bounded as follows:

$$
\overline{\mathcal{T}}_{12} \lesssim \log \left(\frac{P \log K}{2}\right)-2 \sqrt{\mathbb{E}\left\{\log \alpha^{-1}\right\}} \sqrt{\log \log \log K}
$$

Proof: Noting $\beta \geq \log (2)+\frac{1}{2}$, from (24), we have $\rho^{\text {opt }}=1$. Hence, using (26) and (28) and noting $v \sim \log K$, we can write

$$
\begin{aligned}
\overline{\mathcal{T}}_{12} & \leq \log (P \log K)-(N+1) \mathbb{E}\left\{\log \left(\alpha^{-1}\right)\right\}-\mathbb{E}\{\beta \mid \mathcal{A}\} \operatorname{Pr}\{\mathcal{A}\} \\
& \stackrel{25}{\lesssim} \log (P \log K)-(N+1) \mathbb{E}\left\{\log \left(\alpha^{-1}\right)\right\}-\mathbb{E}\left\{\log (2)+\frac{\log (1+N R)}{N} \mid \mathcal{A}\right\} \operatorname{Pr}\{\mathcal{A}\} \\
& \stackrel{a}{\lesssim} \log (P \log K)-(N+1) \mathbb{E}\left\{\log \left(\alpha^{-1}\right)\right\} \\
& -\left[\frac{\log \left(\frac{1}{2} \log \log K-\frac{\log (1+N \log (P \log K))}{N}-\log (2)\right)}{N}+\frac{\log N}{N}+\log (2)\right] \operatorname{Pr}\{\mathcal{A}\}
\end{aligned}
$$

where (a) follows from the fact that conditioned on $\mathcal{A}$, we have

$$
\begin{aligned}
R & =R^{*}-\beta \\
& >\frac{1}{2} \log \log K-\frac{\log (1+N R)}{N}-\log (2) \\
& >\frac{1}{2} \log \log K-\frac{\log (1+N \log (P \log K))}{N}-\log (2),
\end{aligned}
$$

The last line results from the fact that $R<\log (P \log K)$ which follows from (22). Substituting (31) in (39), and setting the derivative of $\overline{\mathcal{T}}_{12}$ to zero with respect to $N$, we obtain

$$
N^{\text {opt }} \sim \sqrt{\frac{\log \log \log K}{\mathbb{E}\left\{\log \alpha^{-1}\right\}}}[1+o(1)] .
$$

Substituting (31) and (41) in (39), the result of the lemma follows.

Lemma 3 Assume that $N$ is set as in (41). Then, under condition $\mathcal{A}$, we have $\rho^{\mathrm{opt}}=1$ as $K \rightarrow \infty$.

Proof: If $\rho^{\text {opt }}<1$, then using (24), we have $\beta<\frac{1}{2}+\log (2)$. Noting (34) and (35), we can write

$$
\begin{aligned}
& N^{\mathrm{opt}} \stackrel{\text { 34 }}{>} \log \left(1+\rho^{\mathrm{opt}} N^{\mathrm{opt}} R\right) \\
& \stackrel{35}{>} \log (1+2 R) \\
& \stackrel{\text { 26) }}{=} \log \left(1+2\left(R^{*}-\beta\right)\right) \\
& >\quad \log \left(2 R^{*}-2 \log (2)\right) \\
& \stackrel{27}{\sim} \log \log \log K \text {, }
\end{aligned}
$$

which contradicts (41).

Lemma 4 The throughput of Strategy I is lower-bounded as follows:

$$
\overline{\mathcal{T}}_{1} \gtrsim \log \left(\frac{P \log K}{2}\right)-2 \sqrt{\mathbb{E}\left\{\log \alpha^{-1}\right\} \log \log \log K} .
$$


Proof: Choosing $N=N^{\text {opt }}$ and subsequently replacing $\rho^{\text {opt }}=1$ (Lemma 3), we compute $R$ in (22), under condition $\mathcal{A}$, as follows:

$$
\begin{aligned}
R & =R^{*}-\beta \\
& >\frac{1}{2} \log \log K-\log (2)-\frac{\log \left(1+N^{\text {opt }} R\right)}{N^{\text {opt }}} \\
& >\frac{1}{2} \log \log K-\log (2)-\frac{\log \left(1+N^{\text {opt }} \log \log K\right)}{N^{\text {opt }}} \\
& \sim \frac{1}{2} \log \log K-\sqrt{\left.\mathbb{E}\left\{\log \alpha^{-1}\right\} \log \log \log K\right)}
\end{aligned}
$$

Using (23) and (26), we can lower-bound $\overline{\mathcal{T}}_{1}$ as

$$
\begin{aligned}
\overline{\mathcal{T}}_{1} \geq & \overline{\mathcal{T}}_{1 \mid \mathcal{A}} \operatorname{Pr}\{\mathcal{A}\} \\
= & \mathbb{E}\left\{\left[R^{*}-\log \left(1+\rho^{\mathrm{opt}}\right)-\frac{\log \left(1+\rho^{\mathrm{opt}} N^{\mathrm{opt}} R\right)}{\rho^{\mathrm{opt}} N^{\mathrm{opt}}}\right]\left[1-\frac{1}{1+\rho^{\mathrm{opt}} N^{\mathrm{opt}} R}\right] \mid \mathcal{A}\right\} \times \\
& \operatorname{Pr}\{\mathcal{A}\},
\end{aligned}
$$

where $\overline{\mathcal{T}}_{1 \mid \mathcal{A}}$ denotes the throughput of the system conditioned on $\mathcal{A}$. Since $\mathbb{E}\left\{R^{*}\right\}=\mathbb{E}\left\{R^{*} \mid \mathcal{A}\right\} \operatorname{Pr}\{\mathcal{A}\}+$ $\mathbb{E}\left\{R^{*} \mid \mathcal{A}^{C}\right\} \operatorname{Pr}\left\{\mathcal{A}^{C}\right\}$, and $\mathbb{E}\left\{R^{*} \mid \mathcal{A}\right\}>\mathbb{E}\left\{R^{*} \mid \mathcal{A}^{C}\right\}$, it follows that $\mathbb{E}\left\{R^{*} \mid \mathcal{A}\right\} \geq \mathbb{E}\left\{R^{*}\right\}$. Having this fact, noting Lemma (3) and using (31), (44) and (45), we can write

$$
\begin{aligned}
\overline{\mathcal{T}}_{1} \geq & \left(\mathbb{E}\left\{R^{*}\right\}-\log (2)-\mathbb{E}\left\{\frac{\log \left(1+N^{\text {opt }} \log \left(\frac{P \log K}{2}\right)\right)}{N^{\text {opt }}} \mid \mathcal{A}\right\}\right) \times \\
& \left(1-\frac{1}{1+N^{\text {opt }}\left(\frac{1}{2} \log \log K-\sqrt{\left.\mathbb{E}\left\{\log \alpha^{-1}\right\} \log \log \log K\right)}\right)}\right)\left(1-e^{-\frac{\log \log K}{2\left(N^{\text {opt }}+1\right)}}\right) \\
\sim & \log \left(\frac{P \log K}{2}\right)-2 \sqrt{\mathbb{E}\left\{\log \alpha^{-1}\right\} \log \log \log K} .
\end{aligned}
$$

The Proof of Theorem 2: Lemma (1) and Lemma (2) provide upper-bounds on two complementary cases where $\rho^{\mathrm{opt}}$ of the selected user is either less than 1 or equal to 1 in (32) and (38), respectively. Lemma (4) lower-bounds the throughput of the system as in (43). Comparing (32), (38) and (43), we conclude the result of the theorem.

Remark 1- To prove Theorem 2, we utilize the distribution function of $\alpha$ to calculate $\operatorname{Pr}(\mathcal{A})$. The value of $\operatorname{Pr}(\mathcal{A})$ is used in (37), (39) and (46). For $\operatorname{Pr}(\mathcal{A})=1-o\left(\frac{1}{\log \log K}\right)$, (37), (39) and (46) are valid. Therefore, the assumption of uniform distribution for the correlation coefficients can be relaxed if $\operatorname{Pr}(\mathcal{A})=$ $1-o\left(\frac{1}{\log \log K}\right)$.

\section{B. Strategy II: SNR-based scheduling with adaptive codeword length}

In this scheme, the BS transmits to the user with the maximum initial fading gain. The rate and codeword length are selected to maximize the corresponding throughput.

Theorem 3 Assuming $K \rightarrow \infty$, the asymptotic throughput of the system under Strategy II scales as follows:

$$
\overline{\mathcal{T}}_{2} \sim \log \left(\frac{P \log K}{2}\right)-2 \mathbb{E}\left\{\sqrt{\log \left(\alpha^{-1}\right)}\right\} \sqrt{\log \log \left(\frac{P \log K}{2}\right)} .
$$


Proof: The throughput of the system can be written as

$$
\overline{\mathcal{T}}_{2}=\overline{\mathcal{T}}_{2 \mid \mathcal{B}} \operatorname{Pr}\{\mathcal{B}\}+\overline{\mathcal{T}}_{2 \mid \mathcal{B}^{C}} \operatorname{Pr}\left\{\mathcal{B}^{C}\right\}
$$

where $\mathcal{B}$ represents the event that $\rho^{\text {opt }}=1, \overline{\mathcal{T}}_{2 \mid \mathcal{B}}$ denotes the throughput conditioned on $\mathcal{B}$, and $\overline{\mathcal{T}}_{2 \mid \mathcal{B}^{C}}$ is the throughput of the system conditioned on $\mathcal{B}^{C}$, the complement of $\mathcal{B}$. Using (17), we can write

$$
\overline{\mathcal{T}}_{2 \mid \mathcal{B}}=\mathbb{E}\left\{\log \left(\frac{P v}{2}\right)-2 \sqrt{\log \left(\alpha^{-1}\right) \log \log \left(\frac{P v}{2}\right)}\left(1+O\left(\frac{\log \log \log (v)}{\log \log (v)}\right)\right) \mid \mathcal{B}\right\},
$$

where $v=\max _{1 \leq k \leq K} v_{k}$, and $\alpha$ is the channel correlation coefficient of the selected user. Noting that $v \sim \log K+O(\log \log K)$ with probability one, and $v$ and $\alpha$ are independent, we have

$$
\begin{aligned}
\overline{\mathcal{T}}_{2 \mid \mathcal{B}} \sim & \log \left(\frac{P \log K}{2}\right)-2 \mathbb{E}\left\{\sqrt{\log \left(\alpha^{-1}\right)} \mid \mathcal{B}\right\} \sqrt{\log \log \left(\frac{P \log K}{2}\right)} \times \\
& \left(1+O\left(\frac{\log \log \log \log K}{\log \log \log K}\right)\right) .
\end{aligned}
$$

Using (16) and (18), we can write

$$
\begin{aligned}
\mathcal{B} & \equiv N^{\mathrm{opt}} \log \left(\alpha^{-1}\right) \geq \frac{1}{2} \\
& \cong \sqrt{\log \left(\alpha^{-1}\right)} \sqrt{\log \log \log K} \geq \frac{1}{2}
\end{aligned}
$$

Uniform distribution for $\alpha$ results in exponential distribution for $X \triangleq \log \left(\alpha^{-1}\right)$, i.e., $f_{X}(x)=e^{-x} u(x)$. Let us define $\epsilon \triangleq \frac{1}{4 \log \log \log K} \cdot \operatorname{Pr}\{\mathcal{B}\}$ can be derived as follows:

$$
\begin{aligned}
\operatorname{Pr}\{\mathcal{B}\} & =\operatorname{Pr}\left\{\log \left(\alpha^{-1}\right) \geq \epsilon\right\} \\
& =e^{-\epsilon}
\end{aligned}
$$

Using (52), we have

$$
\begin{aligned}
\mathbb{E}\left\{\sqrt{\log \left(\alpha^{-1}\right)} \mid \mathcal{B}\right\} & =\frac{\int_{\mathcal{B}} \sqrt{x} e^{-x} d x}{\operatorname{Pr}\{\mathcal{B}\}} \\
& =\frac{\int_{\epsilon}^{\infty} \sqrt{x} e^{-x} d x}{\operatorname{Pr}\{\mathcal{B}\}} \\
& \sim \frac{\mathbb{E}\left\{\sqrt{\log \left(\alpha^{-1}\right)}\right\}-\epsilon \sqrt{\epsilon} e^{-\epsilon}}{e^{-\epsilon}} \\
& \sim \mathbb{E}\left\{\sqrt{\log \left(\alpha^{-1}\right)}\right\}(1+O(\epsilon)) .
\end{aligned}
$$

Similarly, we can write

$$
\begin{aligned}
\mathbb{E}\left\{\sqrt{\log \left(\alpha^{-1}\right)} \mid \mathcal{B}^{C}\right\} & =\frac{\int_{0}^{\epsilon} \sqrt{x} e^{-x} d x}{\operatorname{Pr}\left\{\mathcal{B}^{C}\right\}} \\
& \sim \frac{\epsilon \sqrt{\epsilon} e^{-\epsilon}}{1-e^{-\epsilon}} \\
& \sim O(\sqrt{\epsilon})
\end{aligned}
$$


Using (14), $\overline{\mathcal{T}}_{2 \mid \mathcal{B}^{C}}$ can be written as

$$
\begin{aligned}
& \overline{\mathcal{T}}_{2 \mid \mathcal{B}^{C}}=\mathbb{E}\left\{\left(\log \left(\frac{P v}{1+\rho^{\mathrm{opt}}}\right)-\left(2 N^{\mathrm{opt}}+1\right) \log \left(\alpha^{-1}\right)\right)\left(1-\frac{1}{1+\rho^{\mathrm{opt}} N^{\mathrm{opt}} R^{\mathrm{opt}}}\right) \mid \mathcal{B}^{C}\right\} \\
& \gtrsim \mathbb{E}\left\{\left(\log \left(\frac{P v}{1+\rho^{\mathrm{opt}}}\right)-2 \frac{\rho^{\mathrm{opt}}}{1+\rho^{\mathrm{opt}}}-\log \left(\alpha^{-1}\right)\right)\left(1-\frac{1}{1+\rho^{\mathrm{opt}} N^{\mathrm{opt}} R^{\mathrm{opt}}}\right) \mid \mathcal{B}^{C}\right\} \\
& \gtrsim \mathbb{E}\left\{\left(\log \left(\frac{P v}{1+\rho^{\mathrm{opt}}}\right)-2 \frac{\rho^{\mathrm{opt}}}{1+\rho^{\mathrm{opt}}}-\log \left(\alpha^{-1}\right)\right)\left(1-\frac{1}{1+R^{\mathrm{opt}}}\right) \mid \mathcal{B}^{C}\right\} \\
& \gtrsim \log \left(\frac{P \log K}{2}\right)-\mathbb{E}\left\{\log \left(\alpha^{-1}\right) \mid \mathcal{B}^{C}\right\}-2 \\
& \stackrel{54}{\gtrsim} \log \left(\frac{P \log K}{2}\right)-2-O(\sqrt{\epsilon})
\end{aligned}
$$

where (a) follows from (16) which implies $N^{\mathrm{opt}} \log \left(\alpha^{-1}\right)=\frac{\rho^{\mathrm{opt}}}{1+\rho^{\mathrm{opt}}}$ conditioned on $\mathcal{B}^{C}$ and $(b)$ results from the following inequality:

$$
\begin{aligned}
\rho^{\mathrm{opt}} N^{\mathrm{opt}} R^{\mathrm{opt}} & \stackrel{16}{=} \frac{R^{\mathrm{opt}}\left(N^{\mathrm{opt}}\right)^{2} \log \left(\alpha^{-1}\right)}{1-N^{\mathrm{opt}} \log \left(\alpha^{-1}\right)} \\
& \geq R^{\mathrm{opt}}\left(N^{\mathrm{opt}}\right)^{2} \log \left(\alpha^{-1}\right) \\
& \stackrel{13}{=} R^{\mathrm{opt}} \frac{\log \left(1+\rho^{\mathrm{opt}} N^{\mathrm{opt}} R^{\mathrm{opt}}\right)}{\rho^{\mathrm{opt}}} \\
& \stackrel{a}{\geq} R^{\mathrm{opt}} \log \left(1+N^{\mathrm{opt}} R^{\mathrm{opt}}\right) \\
& \gtrsim R^{\mathrm{opt}}
\end{aligned}
$$

where (a) follows from the fact that $\frac{\log \left(1+\rho^{\mathrm{opt}} N^{\mathrm{opt}} R^{\mathrm{opt}}\right)}{\rho^{\mathrm{opt}}}$ is a decreasing function of $\rho^{\mathrm{opt}}$. Moreover, using (14) and noting that $v \sim \log K$ with probability one, we have

$$
\begin{aligned}
\overline{\mathcal{T}}_{2 \mid \mathcal{B}^{C}} & \leq \mathbb{E}\left\{\log (P v) \mid \mathcal{B}^{C}\right\} \\
& \lesssim \log (P \log K)
\end{aligned}
$$

Combining (55) and (57), we have

$$
\log \left(\frac{P \log K}{2}\right)-2-O(\sqrt{\epsilon}) \lesssim \overline{\mathcal{T}}_{2 \mid \mathcal{B}^{C}} \lesssim \log \left(\frac{P \log K}{2}\right)+\log (2)
$$

Substituting (50) and (58) in (48) and noting (52) and (53), after some manipulations, we have

$$
\begin{aligned}
\overline{\mathcal{T}}_{2} & \sim\left(\log \left(\frac{P \log K}{2}\right)-2 \mathbb{E}\left\{\sqrt{\log \left(\alpha^{-1}\right)} \mid \mathcal{B}\right\} \sqrt{\log \log \left(\frac{P \log K}{2}\right)}\right) \operatorname{Pr}\{\mathcal{B}\} \\
& +\left(\left(\frac{P \log K}{2}\right)+\Omega(1)\right) \operatorname{Pr}\left\{\mathcal{B}^{C}\right\} \\
& \sim \log \left(\frac{P \log K}{2}\right)-2 \mathbb{E}\left\{\sqrt{\log \left(\alpha^{-1}\right)}\right\} \sqrt{\log \log \left(\frac{P \log K}{2}\right)} \\
& +2 \mathbb{E}\left\{\sqrt{\log \left(\alpha^{-1}\right)}\right\} \sqrt{\log \log \left(\frac{P \log K}{2}\right)} \epsilon \sqrt{\epsilon} e^{-\epsilon}+\Omega(1) O(\sqrt{\epsilon}) \\
& \sim \log \left(\frac{P \log K}{2}\right)-2 \mathbb{E}\left\{\sqrt{\log \left(\alpha^{-1}\right)}\right\} \sqrt{\log \log \left(\frac{P \log K}{2}\right)}+O(\sqrt{\epsilon})
\end{aligned}
$$


which completes the proof of Theorem 3.

Remark 1- To prove Theorem 3, we used the following properties:

$$
\begin{array}{r}
\mathbb{E}\left\{\sqrt{\log \left(\alpha^{-1}\right)} \mid \mathcal{B}\right\} \sim \mathbb{E}\left\{\sqrt{\log \left(\alpha^{-1}\right)}\right\}(1+O(\epsilon)) \\
\mathbb{E}\left\{\sqrt{\log \left(\alpha^{-1}\right) \mid} \mid \mathcal{B}^{\mathcal{C}}\right\} \sim O(\sqrt{\epsilon}) \\
\operatorname{Pr}\left\{\mathcal{B}^{C}\right\} \sim O(\epsilon),
\end{array}
$$

where $\epsilon \sim \frac{1}{\log \log \log K}$. The theorem is valid for any distribution function of $\alpha$ that satisfies the above properties.

Remark 2- Since $\mathbb{E}\{\sqrt{x}\} \leq \sqrt{\mathbb{E}\{x\}}$, for $x>0$, it is concluded that the achievable rate of Strategy II is higher than that of Strategy I. More precisely,

$$
\overline{\mathcal{T}}_{2}-\overline{\mathcal{T}}_{1} \sim 2\left(\sqrt{\mathbb{E}\left\{\log \left(\alpha^{-1}\right)\right\}}-\mathbb{E}\left\{\sqrt{\log \left(\alpha^{-1}\right)}\right\}\right) \sqrt{\log \log \log K}
$$

For the case of uniform distribution for $\alpha$, we have

$$
\overline{\mathcal{T}}_{2}-\overline{\mathcal{T}}_{1} \sim 0.228 \sqrt{\log \log \log K}
$$

Remark 3- Although $\lim _{K \rightarrow \infty} \frac{\overline{\mathcal{T}}_{1}}{\overline{\mathcal{T}}_{\max }}=\lim _{K \rightarrow \infty} \frac{\overline{\mathcal{T}}_{2}}{\overline{\mathcal{T}}_{\max }}=1$, where $\overline{\mathcal{T}}_{\text {max }} \sim \log (P \log K)$ is the maximum achievable throughput for a quasi-static fading channel [21], there exists a gap of $\Omega(\sqrt{\log \log \log K})$ between the achievable throughput of Strategies I and II, and the maximum throughput. As we show later, this gap is due to the fact that the channel correlation coefficients of the users are not considered in the scheduling. In fact, this gap approaches zero by exploiting the channel correlation, which is discussed in Strategy III.

\section{Strategy III: Scheduling based on both SNR and channel correlation coefficient with adaptive codeword length}

To maximize the throughput of the system, the user which maximizes the expression in (14) should be serviced. Here, for simplicity of analysis, we propose a sub-optimum scheduling that considers the effect of both SNR and channel correlation in the user selection. In this strategy, each user is required to feed back its initial fading gain only if it is greater than a pre-determined threshold $\sqrt{\Theta}$, where $\Theta$ is a function of the number of users. Among these users, the BS selects the one with the maximum channel correlation coefficient. The data rate and codeword length are selected to maximize the corresponding throughput. The following theorem gives the system throughput under this strategy.

Theorem 4 Using Strategy III, with $\Theta$ satisfying

$$
\log K-o(\log K) \lesssim \Theta \lesssim \log K-\log \log K-\omega(1),
$$

the throughput of the system scales as

$$
\overline{\mathcal{T}}_{3} \gtrsim \log (P \log K)-o(1)
$$

Proof: Define $\mathcal{S} \triangleq\left\{k \mid v_{k} \geq \Theta\right\}$ and $\alpha_{\max } \triangleq \max _{k \in \mathcal{S}} \alpha_{k}$. Let $v$ be the squared initial fading gain of the user corresponding to $\alpha_{\max }$. We define the event $\mathcal{G}$ as follows:

$$
\mathcal{G} \equiv N^{\text {opt }} \log \left(\alpha_{\text {max }}^{-1}\right) \sim o(1),
$$

where $N^{\text {opt }}$ is the corresponding codeword length as computed from (13). Using (19) and (65), we can write

$$
\overline{\mathcal{T}}_{3} \geq \operatorname{Pr}\{\mathcal{G}\} \mathbb{E}\left\{\mathcal{T}_{3}\left(v, \alpha_{\text {max }}\right) \mid \mathcal{G}\right\}
$$


where following (19),

$$
\begin{aligned}
\mathbb{E}\left\{\mathcal{T}_{3}\left(v, \alpha_{\max }\right) \mid \mathcal{G}\right\}= & \mathbb{E}\left\{\log (P v)-2 \sqrt[3]{\log \left(\alpha_{\max }^{-1}\right)}\right. \\
& \left.\sqrt[3]{\log \log (P v)}\left[1+O\left(\frac{\log \log \log (v)}{\log \log (v)}\right)\right]-o(1) \mid \mathcal{G}\right\}
\end{aligned}
$$

Noting that $\left\{\mathcal{T}_{3}\left(v, \alpha_{\max }\right) \mid \mathcal{G}\right\}$ in (67) is an increasing function of $v$, we have

$$
\begin{aligned}
\mathbb{E}\left\{\mathcal{T}_{3}\left(v, \alpha_{\max }\right) \mid \mathcal{G}\right\} \geq & \log (P \Theta)-2 \mathbb{E}\left\{\sqrt[3]{\log \left(\alpha_{\max }^{-1}\right)} \mid \mathcal{G}\right\} \\
& \sqrt[3]{\log \log (P \Theta)}\left[1+O\left(\frac{\log \log \log (\Theta)}{\log \log (\Theta)}\right)\right]-o(1) \\
\geq & \log (P \Theta)-2 \mathbb{E}\left\{\sqrt[3]{\log \left(\alpha_{\max }^{-1}\right)}\right\} \\
& \sqrt[3]{\log \log (P \Theta)}\left[1+O\left(\frac{\log \log \log (\Theta)}{\log \log (\Theta)}\right)\right]-o(1) \\
\geq & \log (P \Theta)-2 \sqrt[3]{\mathbb{E}\left\{\log \left(\alpha_{\max }^{-1}\right)\right\}} \\
& \sqrt[3]{\log \log (P \Theta)}\left[1+O\left(\frac{\log \log \log (\Theta)}{\log \log (\Theta)}\right)\right]-o(1),
\end{aligned}
$$

where (a) follows from the fact that $\mathbb{E}\left\{\sqrt[3]{\log \left(\alpha_{\max }^{-1}\right)} \mid \mathcal{G}\right\} \leq \mathbb{E}\left\{\sqrt[3]{\log \left(\alpha_{\max }^{-1}\right)}\right\}$ and (b) results from the convexity of cube root. For large values of $K, \mathbb{E}\left\{\log \left(\alpha_{\max }^{-1}\right)\right\}$ can be approximated as follows (See Appendix B):

$$
\mathbb{E}\left\{\log \left(\alpha_{\max }^{-1}\right)\right\} \simeq \frac{1}{K e^{-\Theta}}\left(1+O\left(\frac{1}{K e^{-\Theta}}\right)\right)+e^{-K e^{-\Theta}}(\Theta-\log K) .
$$

Noting (69), for values of $\Theta$ satisfying (63), we have

$$
\mathbb{E}\left\{\log \left(\alpha_{\max }^{-1}\right)\right\} \log \log (\Theta) \sim o(1) .
$$

Using (68) and (70), we can write

$$
\mathbb{E}\left\{\mathcal{T}_{3}\left(v, \alpha_{\max }\right) \mid \mathcal{G}\right\} \geq \log (P \log K)-o(1) .
$$

To compute $\operatorname{Pr}\{\mathcal{G}\}$ defined in 65 , we use Chebychev inequality.

$$
\operatorname{Pr}\{|\mathcal{Z}-\mathbb{E}\{\mathcal{Z}\}|<\sqrt{\sqrt[3]{\log K} \operatorname{var}\{\mathcal{Z}\}}\}>1-\frac{1}{\sqrt[3]{\log K}}
$$

where $\mathcal{Z}=N^{\text {opt }} \log \left(\alpha_{\max }^{-1}\right)$. Noting (20), (63) and (69), we have

$$
\begin{aligned}
& \mathbb{E}\left\{N^{\text {opt }} \log \left(\alpha_{\max }^{-1}\right)\right\} \stackrel{\sqrt[20]{\leq}}{\leq} \mathbb{E}\left\{\sqrt[3]{\log \left(\alpha_{\max }^{-1}\right) \log \log \left(P v_{\max }\right)}\right\} \\
& =\mathbb{E}\left\{\sqrt[3]{\log \left(\alpha_{\max }^{-1}\right)}\right\} \mathbb{E}\left\{\sqrt[3]{\log \log \left(P v_{\max }\right)}\right\} \\
& \lesssim \sqrt[3]{\mathbb{E}\left\{\log \left(\alpha_{\max }^{-1}\right)\right\}} \sqrt[3]{\log \log (P \log K)} \\
& \stackrel{70}{=} o(1)
\end{aligned}
$$


where $(a)$ follows from the fact the $v_{\max } \sim \log (K)$ with probability one. Also, noting (20), (63) and (69), we have

$$
\begin{aligned}
\operatorname{var}\left\{N^{\text {opt }} \log \left(\alpha_{\text {max }}^{-1}\right)\right\} & =\operatorname{var}\left\{\sqrt[3]{\log \left(\alpha_{\text {max }}^{-1}\right) \log \log (P v)}\right\} \\
\leq & \mathbb{E}\left\{\left(\log \left(\alpha_{\text {max }}^{-1}\right) \log \log (P v)\right)^{\frac{2}{3}}\right\} \\
\leq & \left(\mathbb{E}\left\{\log \left(\alpha_{\max }^{-1}\right)\right\}\right)^{\frac{2}{3}}(\mathbb{E}\{\log \log (P v)\})^{\frac{2}{3}} \\
& \stackrel{63}{\lesssim}, a\left(\frac{1}{(\log K)^{\frac{2}{3}}}\right) O\left((\log \log \log K)^{\frac{2}{3}}\right) \\
& O\left(\left(\frac{\log \log \log K}{\log K}\right)^{\frac{2}{3}}\right)
\end{aligned}
$$

where $(a)$ follows from the fact that $v \sim \log K+O(\log \log K)$ with probability one. Substituting (73) and (74) in (72), we have

$$
\operatorname{Pr}\left\{\left|N^{\text {opt }} \log \left(\alpha_{\max }^{-1}\right)-o(1)\right|<O\left(\left(\frac{\log \log \log K}{\sqrt{\log K}}\right)^{\frac{1}{3}}\right)\right\}>1-\frac{1}{\sqrt[3]{\log K}}
$$

Noting (66), (71), and (75), the result of the theorem follows.

Remark 1- The uniform distribution of the correlation coefficients is not a necessary condition for Theorem 4. In fact, Theorem 4 is valid if $\operatorname{Pr}\{\mathcal{G}\} \sim 1-o\left(\frac{1}{\log \log K}\right) . \operatorname{Pr}\{\mathcal{G}\}$ can be written as

$$
\begin{aligned}
\operatorname{Pr}\left\{N^{\mathrm{opt}} \log \left(\alpha_{\text {max }}^{-1}\right)<g(K)\right\} & =\operatorname{Pr}\left\{\sqrt[3]{\log \left(\alpha_{\max }^{-1}\right) \log \log (P \log K)}<g(K)\right\} \\
& =\operatorname{Pr}\left\{\alpha_{\max }>e^{\frac{-g(K)^{3}}{\log \log (P \log K)}}\right\} \\
& =1-\left(F_{\alpha}\left(e^{\frac{-g(K)^{3}}{\log \log (P \log K)}}\right)\right)^{K}
\end{aligned}
$$

where $g(K)$ satisfies $g(K) \sim o(1)$. Noting (76), there must exist a function $g(K)$ such that $F_{\alpha}\left(e^{\frac{-g(K)^{3}}{\log \log (P \log K)}}\right) \sim$ $1-\omega\left(\frac{\log \log \log K}{K}\right)$ to satisfy $\operatorname{Pr}\{\mathcal{G}\} \sim 1-o\left(\frac{1}{\log \log K}\right)$. Hence, there exists a larger class of distributions that satisfy the requirements for this theorem.

\section{CONCLUSION}

A multiuser downlink communication over a time-correlated fading channel has been considered. We have proposed three scheduling schemes in order to maximize the throughput of the system. Assuming a large number of users in the system, we show that using SNR-based scheduling, a gap of $\Omega(\sqrt{\log \log \log K})$ exists between the achievable throughput and the maximum throughput of the system. We propose a simple scheduling, considering both the SNR and channel correlation of the users. We show that the gap between the throughput of the proposed scheme and the maximum throughput of the system approaches zero as the number of users tends to infinity.

\section{Appendix A}

For simplicity, we drop the user index. Noting (6), we have $E_{0}(\rho)=-\frac{1}{N} \log I_{N}$, where

$$
I_{N}=\int_{u_{N}} \ldots \int_{u_{1}} \prod_{i=1}^{N}\left(\frac{1}{1+\frac{P}{1+\rho} u_{i}^{2}}\right)^{\rho} p\left(\mathbf{u} \mid u_{0}\right) d u_{i}
$$

Using (3), we have

$$
I_{N}=\int_{u_{N}} \ldots \int_{u_{1}} \prod_{i=1}^{N} \frac{2 u_{i}}{1-\alpha^{2}} \exp \left\{-\frac{u_{i}^{2}+\alpha^{2} u_{i-1}^{2}}{1-\alpha^{2}}\right\} \mathcal{I}_{0}\left(\frac{2 \alpha u_{i} u_{i-1}}{1-\alpha^{2}}\right)\left(\frac{1}{1+\frac{P}{1+\rho} u_{i}^{2}}\right)^{\rho} d u_{i} .
$$


Substituting $v_{i}=\frac{u_{i}}{u_{0} \sqrt{\left(1-\alpha^{2}\right) / 2}}, 0 \leq i \leq N$, we have

$$
\begin{array}{r}
I_{N}=\int_{v_{N}} \ldots \int_{v_{1}} \prod_{i=1}^{N} u_{0}^{2} v_{i} e^{-\frac{v_{i}^{2}+\alpha^{2} v_{i-1}^{2}}{2 / u_{0}^{2}}} \mathcal{I}_{0}\left(\alpha u_{0}^{2} v_{i} v_{i-1}\right) f\left(v_{i}\right) d v_{i} \\
=\int_{v_{N}} \ldots \int_{v_{1}} \prod_{i=1}^{N} u_{0}^{2} v_{i} e^{-\frac{\left(v_{i}-\alpha v_{i-1}\right)^{2}}{2 / u_{0}^{2}}} e^{-\alpha u_{0}^{2} v_{i} v_{i-1}} \mathcal{I}_{0}\left(\alpha u_{0}^{2} v_{i} v_{i-1}\right) f\left(v_{i}\right) d v_{i},
\end{array}
$$

where,

$$
f\left(v_{i}\right)=\left(\frac{1}{1+\frac{P u_{0}^{2}\left(1-\alpha^{2}\right)}{2(1+\rho)} v_{i}^{2}}\right)^{\rho} .
$$

For large values of $u_{0}$, we evaluate the following integral.

$$
\begin{aligned}
I & =u_{0}^{2} v e^{-\frac{(v-\mu)^{2}}{2 / u_{0}^{2}}} e^{-u_{0}^{2} v \mu} \mathcal{I}_{0}\left(u_{0}^{2} v \mu\right) \varphi(v) \\
& =\int_{0}^{\infty} g(v) \frac{1}{\sqrt{2 \pi / u_{0}^{2}}} e^{-\frac{(v-\mu)^{2}}{2 / u_{0}^{2}}} d v
\end{aligned}
$$

where $g(v) \triangleq \sqrt{2 \pi} v u_{0} \mathcal{I}_{0}\left(u_{0}^{2} v \mu\right) e^{-u_{0}^{2} v \mu} \varphi(v)$ and $\varphi(v)$ is differentiable and satisfies $0 \leq \varphi(v) \leq 1$ and $\varphi(v) \sim O\left(\frac{1}{u_{0}^{\rho}}\right)$. Noting that [22]

$$
\mathcal{I}_{0}(z) e^{-z} \sqrt{2 \pi z}=1+O\left(\frac{1}{z}\right), \quad z \gg 1
$$

it is easy to show that $g^{(n)}(\mu)$ is bounded for $\mu \geq 0$ and $n \geq 1$. Using Taylor series of $g(v)$ about $\mu$, we have

$$
\begin{aligned}
I & =\int_{0}^{\infty}\left(g(\mu)+\sum_{n=1}^{\infty} \frac{g^{(n)}(\mu)}{n !}(v-\mu)^{n}\right) \frac{1}{\sqrt{2 \pi / u_{0}^{2}}} e^{-\frac{(v-\mu)^{2}}{2 / u_{0}^{2}}} d v \\
& =g(\mu)\left(1-Q\left(\mu u_{0}\right)\right)+\int_{0}^{\infty} \sum_{n=1}^{\infty} \frac{g^{(n)}(\mu)}{n !}(v-\mu)^{n} \frac{1}{\sqrt{2 \pi / u_{0}^{2}}} e^{-\frac{(v-\mu)^{2}}{2 / u_{0}^{2}}} d v \\
& =g(\mu)\left(1-Q\left(\mu u_{0}\right)\right)+\int_{\left[\mu-\frac{1}{\sqrt{u_{0}}}\right]+}^{\mu+\frac{1}{\sqrt{u_{0}}}} \sum_{n=1}^{\infty} \frac{g^{(n)}(\mu)}{n !}(v-\mu)^{n} \frac{1}{\sqrt{2 \pi / u_{0}^{2}}} e^{-\frac{(v-\mu)^{2}}{2 / u_{0}^{2}}} d v+\varepsilon \\
& =g(\mu)\left(1-Q\left(\mu u_{0}\right)\right)+O\left(\frac{g^{\prime}(\mu)}{\sqrt{u_{0}}}\right)+\varepsilon .
\end{aligned}
$$


where $\varepsilon$ can be bounded as follows:

$$
\begin{aligned}
\varepsilon & \stackrel{a}{\leq} \int_{0}^{\left[\mu-\frac{1}{\sqrt{u_{0}}}\right]^{+}} \frac{g(v)}{\sqrt{2 \pi / u_{0}^{2}}} e^{-\frac{(v-\mu)^{2}}{2 / u_{0}^{2}}} d v+\int_{\mu+\frac{1}{\sqrt{u_{0}}}}^{\infty} \frac{g(v)}{\sqrt{2 \pi / u_{0}^{2}}} e^{-\frac{(v-\mu)^{2}}{2 / u_{0}^{2}}} d v \\
& \leq \sqrt{2 \pi} u_{0} \int_{0}^{\left[\mu-\frac{1}{\sqrt{u_{0}}}\right]^{+}} \frac{v}{\sqrt{2 \pi / u_{0}^{2}}} e^{-\frac{(v-\mu)^{2}}{2 / u_{0}^{2}}} d v+\sqrt{2 \pi} u_{0} \int_{\mu+\frac{1}{\sqrt{u_{0}}}}^{\infty} \frac{v}{\sqrt{2 \pi / u_{0}^{2}}} e^{-\frac{(v-\mu)^{2}}{2 / u_{0}^{2}}} d v \\
& \leq 2 \sqrt{2 \pi} u_{0} \int_{\mu+\frac{1}{\sqrt{u_{0}}}}^{\infty} \frac{v}{\sqrt{2 \pi / u_{0}^{2}}} e^{-\frac{(v-\mu)^{2}}{2 / u_{0}^{2}}} d v \\
& =2 \sqrt{2 \pi} u_{0}\left(Q\left(\sqrt{u_{0}}\right)\left(\mu+\frac{1}{\sqrt{u_{0}}}\right)+\int_{\sqrt{u_{0}}}^{\infty} Q(z) d z\right) \\
& \leq 2 \sqrt{2 \pi} u_{0}\left(\left(\mu+\frac{1}{\sqrt{u_{0}}}\right) e^{-\frac{u_{0}}{2}}+\int_{\sqrt{u_{0}}}^{\infty} e^{-\frac{z^{2}}{2}} d z\right) \\
& \leq 2 \sqrt{2 \pi} e^{-\frac{u_{0}}{2}} u_{0}\left(\left(\mu+\frac{1}{\sqrt{u_{0}}}\right)+\sqrt{2 \pi}\right) \\
& \leq O\left(u_{0} e^{-\frac{u_{0}}{2}}\right)
\end{aligned}
$$

where (a) results from the fact that $g(\mu) \geq 0$, (b) is valid because $\mathcal{I}_{0}(\mu z) e^{-\mu z} \leq 1$ for $\mu \geq 0$ and $z \geq 0$, and $(c)$ and $(d)$ follow from the fact that $Q(z) \triangleq \frac{1}{\sqrt{2 \pi}} \int_{z}^{\infty} e^{-t^{2} / 2} d t \leq e^{-z^{2} / 2}$. Moreover, using (82), we can write

$$
\begin{aligned}
g(\mu) & =\varphi(\mu) \sqrt{2 \pi} \mu u_{0} \mathcal{I}_{0}\left(u_{0}^{2} \mu^{2}\right) e^{-u_{0}^{2} \mu^{2}} \\
& =\varphi(\mu)\left(1+O\left(\frac{1}{u_{0}^{2}}\right)\right) .
\end{aligned}
$$

Also, using (82) and noting $\varphi(v) \sim O\left(\frac{1}{u_{0}^{\rho}}\right)$, we have

$$
\begin{array}{r}
g(v)=\varphi(v) \sqrt{\frac{v}{\mu}}\left(1+O\left(\frac{1}{u_{0}^{2}}\right)\right) \\
\Rightarrow O\left(g^{\prime}(v)\right)=O\left(\frac{\varphi(v)}{2 \sqrt{v \mu}}+\varphi^{\prime}(v) \sqrt{\frac{v}{\mu}}\right) \\
\Rightarrow O\left(g^{\prime}(\mu)\right)=O(\varphi(\mu)) .
\end{array}
$$

Using (83), (84), (85) and (86), we have

$$
\begin{aligned}
I & =\varphi(\mu)\left(1+O\left(\frac{1}{\sqrt{u_{0}}}\right)\right)+O\left(u_{0} e^{-\frac{u_{0}}{2}}\right) \\
& \stackrel{a}{=} \varphi(\mu)\left(1+O\left(\frac{1}{\sqrt{u_{0}}}\right)\right),
\end{aligned}
$$


where (a) follows from the fact that $\varphi(\mu)=O\left(\frac{1}{u_{0}^{\rho}}\right)$. Applying (87) in (79), we have

$$
\begin{aligned}
I_{N}= & \int_{v_{N-1}} \ldots \int_{v_{1}} f\left(\alpha v_{N-1}\right)\left(1+O\left(\frac{1}{\sqrt{u_{0}}}\right)\right)\left(1-Q\left(\alpha v_{N-1} u_{0}\right)\right) \times \\
& \prod_{i=1}^{N-1} u_{0}^{2} v_{i} e^{-\frac{\left(v_{i}-\alpha v_{i-1}\right)^{2}}{2 / u_{0}^{2}}-\alpha u_{0}^{2} v_{i} v_{i-1}} \mathcal{I}_{0}\left(\alpha u_{0}^{2} v_{i} v_{i-1}\right) f\left(v_{i}\right) d v_{i} \\
= & \int_{v_{N-2}} \ldots \int_{v_{1}} f\left(\alpha^{2} v_{N-2}\right) f\left(\alpha v_{N-2}\right)\left(1+O\left(\frac{1}{\sqrt{u_{0}}}\right)\right)^{2}\left(1-Q\left(\alpha^{2} v_{N-2} u_{0}\right)\right) \times \\
& \left(1-Q\left(\alpha v_{N-2} u_{0}\right)\right) \prod_{i=1}^{N-2} u_{0}^{2} v_{i} e^{-\frac{\left(v_{i}-\alpha v_{i-1}\right)^{2}}{2 / u_{0}^{2}}-\alpha u_{0}^{2} v_{i} v_{i-1}} \mathcal{I}_{0}\left(\alpha u_{0}^{2} v_{i} v_{i-1}\right) f\left(v_{i}\right) d v_{i} \\
= & \cdots=\prod_{i=1}^{N} f\left(\alpha^{i} v_{0}\right)\left(1+O\left(\frac{1}{\sqrt{u_{0}}}\right)\right)\left(1-Q\left(\alpha^{i} v_{0} u_{0}\right)\right)
\end{aligned}
$$

Substituting $v_{0}=\frac{1}{\sqrt{\left(1-\alpha^{2}\right) / 2}}$, we have

$$
\begin{aligned}
I_{N} & =\prod_{i=1}^{N} f\left(\frac{\sqrt{2} \alpha^{i}}{\sqrt{\left(1-\alpha^{2}\right)}}\right)\left(1+O\left(\frac{1}{\sqrt{u_{0}}}\right)\right)\left(1-Q\left(\frac{\sqrt{2} \alpha^{i} u_{0}}{\sqrt{\left(1-\alpha^{2}\right)}}\right)\right) \\
& =\prod_{i=1}^{N} f\left(\frac{\sqrt{2} \alpha^{i}}{\sqrt{\left(1-\alpha^{2}\right)}}\right)\left(1+O\left(\frac{1}{\sqrt{u_{0}}}\right)\right)\left(1-O\left(e^{-u_{0}^{2}}\right)\right)
\end{aligned}
$$

Using (80) and (89) and noting $E_{0}(\rho)=-\frac{1}{N} \log I_{N}$, we conclude Theorem 1

\section{ApPENDIX B}

$\mathbb{E}\left\{\log \left(\alpha_{\max }^{-1}\right)\right\}$ can be derived as follows

$$
\mathbb{E}\left\{\log \left(\alpha_{\text {max }}^{-1}\right)\right\}=\sum_{n=1}^{K} \mathbb{E}\left\{\log \left(\alpha_{\text {max }}^{-1}\right)|| \mathcal{S} \mid=n\right\} \operatorname{Pr}\{|\mathcal{S}|=n\} .
$$

Since $\alpha_{k}, k=1, \cdots, K$, are i.i.d. random variables with uniform distribution, we can write

$$
\begin{aligned}
F_{\alpha_{\max }}(\alpha|| \mathcal{S} \mid=n) & =\alpha^{n} \\
\Rightarrow \mathbb{E}\left\{\log \left(\alpha_{\max }^{-1}\right)|| \mathcal{S} \mid=n\right\} & =\int_{0}^{1} \log \left(\alpha^{-1}\right) n \alpha^{n-1} d \alpha \\
& =\frac{1}{n},
\end{aligned}
$$

where $F_{X}($.$) denotes the cumulative density function of the random variable X$. Indeed, $|\mathcal{S}|$ is a binomial random variable with parameters $K$ and $e^{-\Theta}$. (Since $v_{k}=u_{0, k}^{2}$ and $u_{0, k}$ has a Rayleigh distribution, we have $\left.\operatorname{Pr}\left(v_{k} \geq \Theta\right)=e^{-\Theta}\right)$. Hence,

$$
\operatorname{Pr}\{|\mathcal{S}|=n\}=\left(\begin{array}{c}
K \\
n
\end{array}\right) e^{-n \Theta}\left(1-e^{-\Theta}\right)^{K-n} .
$$

Substituting (91) and (92) in (90), we have

$$
\mathbb{E}\left\{\log \left(\alpha_{\max }^{-1}\right)\right\}=\sum_{n=1}^{K}\left(\begin{array}{c}
K \\
n
\end{array}\right) \frac{1}{n} e^{-n \Theta}\left(1-e^{-\Theta}\right)^{K-n} .
$$


Let us define $\lambda(K) \triangleq \sum_{n=1}^{K}\left(\begin{array}{l}K \\ n\end{array}\right) \frac{1}{n} e^{-n \Theta}\left(1-e^{-\Theta}\right)^{K-n}$.

$$
\begin{array}{r}
\lambda(K)=\sum_{n=0}^{K-1}\left(\begin{array}{c}
K \\
n+1
\end{array}\right) \frac{e^{-(n+1) \Theta}}{n+1}\left(1-e^{-\Theta}\right)^{K-n-1} \\
=\left(1-e^{-\Theta}\right) \sum_{n=0}^{K-2}\left(\begin{array}{c}
K-1 \\
n+1
\end{array}\right) \frac{e^{-(n+1) \Theta}}{n+1}\left(1-e^{-\Theta}\right)^{K-n-2}+ \\
e^{-\Theta} \sum_{n=0}^{K-1}\left(\begin{array}{c}
K-1 \\
n
\end{array}\right) \frac{1}{n+1} e^{-n \Theta}\left(1-e^{-\Theta}\right)^{K-n-1} \\
=\left(1-e^{-\Theta}\right) \lambda(K-1)+\frac{1}{K}-\frac{\left(1-e^{-\Theta}\right)^{K}}{K}
\end{array}
$$

Solving the iteration considering $\lambda(1)=e^{-\Theta}$, we derive $\lambda(K)$ which is equal to $\mathbb{E}\left\{\log \left(\alpha_{\max }^{-1}\right)\right\}$.

$$
\mathbb{E}\left\{\log \left(\alpha_{\max }^{-1}\right)\right\}=\sum_{n=1}^{K} \frac{1}{n}\left(1-e^{-\Theta}\right)^{K-n}-\left(1-e^{-\Theta}\right)^{K} \sum_{n=1}^{K} \frac{1}{n} .
$$

For large values of $K$, we can approximate (95) as

$$
\begin{aligned}
\mathbb{E}\left\{\log \left(\alpha_{\text {max }}^{-1}\right)\right\} \cong & \left(1-e^{-\Theta}\right)^{K}\left(\int_{1}^{K} \frac{\left(1-e^{-\Theta}\right)^{-x} d x}{x}-\int_{1}^{K} \frac{d x}{x}\right) \\
\cong & \frac{1}{-K \log \left(1-e^{-\Theta}\right)}\left(1+O\left(\frac{1}{-K \log \left(1-e^{-\Theta}\right)}\right)\right) \\
& +\left(1-e^{-\Theta}\right)^{K}(\Theta-\log K) \\
\simeq & \frac{1}{K e^{-\Theta}}\left(1+O\left(\frac{1}{K e^{-\Theta}}\right)\right)+e^{-K e^{-\Theta}}(\Theta-\log K) .
\end{aligned}
$$

where $(a)$ results from the following approximations [22]:

$$
\begin{array}{r}
\int_{-\tau}^{\infty} \frac{e^{-t}}{t} d t \simeq \frac{e^{\tau}}{\tau}\left(1+O\left(\frac{1}{\tau}\right)\right)-i \pi \quad \tau \gg 1 \\
\int_{-\tau}^{\infty} \frac{e^{-t}}{t} d t \simeq \log (\tau)-i \pi \quad 0<\tau \ll 1
\end{array}
$$

\section{REFERENCES}

[1] P. Viswanath, D.N.C. Tse, R. Laroia, “Opportunistic beamforming using dumb antennas," IEEE Trans. Inform. Theory, vol. 48, pp. 12771294, June 2002.

[2] D. N. C. Tse and S. Hanly, "Multiaccess fading channels: Part i: Polymatroid structure, optimal resource allocation and throughput capacities,", IEEE Trans. Information Theory, vol. 44, p. 27962815, Nov. 1998.

[3] R. Knopp and P. A. Humblet, "Information capacity and power control in single-cell multiuser communications," IEEE ICC'95, vol. 1, pp. 331 - 335, June 1995.

[4] D. Tse, "Optimal power allocation over parallel gaussian channels," in Proc. Int. Symp. on Inform. Theory, p. 27 , June 1997.

[5] R. Agrawal, A. Bedekar, R. La, and V. Subramanian, "A class and channel condition based weighted proportionally fair scheduler," in Teletraffic Engineering in the Internet Era, Proc. Int. Teletraffic Congr., vol. ITC-17, p. 553565, Sept. 2001.

[6] X. Liu, E. K. P. Chong, and N. B. Shroff, "Opportunistic transmission scheduling with resource-sharing constraints in wireless networks," IEEE JSAC, vol. 19, pp. 2053-2064, Oct. 2001.

[7] Y. Liu and E. Knightly, "Opportunistic fair scheduling over multiple wireless channels," in Proc. of 2003 IEEE INFOCOM, p. 11061115, April 2003.

[8] S. Borst and P. Whiting, "Dynamic rate control algorithms for HDR throughput optimization," in Proc. IEEE INFOCOM 2001, vol. 2, pp. 976-985, April 2001.

[9] P. Bender, P. Black, M. Grob, R. Padovani, N. Sindhushayana, and A. Viterbi, "CDMA/HDR: A bandwidth efficient high-speed wireless data service for nomadic users," IEEE Communications Magazine, pp. 70-77, July 2000.

[10] CDMA 2000: High Rate Packet Data Air Interface Specification. Std. TIA/EIA IS-856, Nov. 2000. 
[11] A. Jalali, R. Padovani, and R. Pankaj , "Data throughput of CDMA/HDR: A high efficiency, high data rate personal wireless system," in Proc. IEEE Vehicular Tech. Conference, vol. 3, pp. 1854-1858, May 2000.

[12] X. Qin and R. Berry , "Exploiting multiuser diversity for medium access control in wireless networks," in Proc. IEEE, INFOCOM, pp. 1084-1094, 2003.

[13] S. Shamai and E. Telatar, "Some information theoretic aspects of decentralized power control in multiple access fading channels," in Proc. Inform. Theory and Networking Workshop, 1999.

[14] N. Sharma and L. H. Ozarow , "A study of opportunism for multiple-antenna systems," IEEE Trans. Inform. Theory, vol. 51, pp. 1804 - 1814, May 2005.

[15] M. Grossglauser and D. Tse, "Mobility increases the capacity of ad-hoc wireless networks," in Proc. INFOCOM 2001, vol. 3, pp. 13601369,2001

[16] A. N. Trofimov, "Convolutional codes for channels with fading," in Proc. Inform Transmission, vol. 27, pp. 155-165, Oct. 1991.

[17] R. G. Galager, Information theory and Reliable communication. J. Wiley, New York, 1968.

[18] R. A. Berry and R. G. Gallager, "Communication over fading channels with delay constraints," IEEE Trans. Inform. Theory, vol. 48, pp. 1135 - 1149, May 2002.

[19] E. Telatar and R. G. Gallager , "Combining queueing theory with information theory for multiaccess," IEEE J. Select. Areas Commun., vol. 13, pp. 963-969, May 1995.

[20] G. Kaplan and S. Shamai , "Achievable performance over the correlated rician channel," IEEE Trans. Commun., vol. 42, pp. 2967 2978, Nov. 1994.

[21] M. Sharif and B. Hassibi, "On the capacity of mimo broadcast channels with partial side information," IEEE Trans. Inform. Theory, vol. 51, pp. $506-522$, Feb. 2005.

[22] Y. L. Luke, The special functions and their approximations. New York, Academic Press, 1969. 